\title{
Lack of Association Between Toxoplasma gondii Infection and Diabetes Mellitus: A Matched Case-Control Study in a Mexican Population
}

\author{
Cosme Alvarado-Esquivel ${ }^{\mathrm{a}, \mathrm{f}}$, Nayely Loera-Moncivais ${ }^{\mathrm{a}}$, Jesus Hernandez-Tinoco ${ }^{\mathrm{b}}$, \\ Luis Francisco Sanchez-Anguiano ${ }^{\mathrm{b}}$, Guillermina Hernandez-Madrid ${ }^{c}$, Elizabeth Rabago-Sanchez ${ }^{\mathrm{a}, \mathrm{d}}$, \\ Maria Magdalena Centeno-Tinoco ${ }^{\mathrm{e}}$, Ada A. Sandoval-Carrillo ${ }^{\mathrm{b}}$, Jose M. Salas-Pacheco ${ }^{\mathrm{b}}$, \\ Oscar Vladimir Campos-Moreno ${ }^{\mathrm{c}}$, Elizabeth Irasema Antuna-Salcido ${ }^{\mathrm{b}}$
}

\begin{abstract}
Background: Very little is known about the association between infection with Toxoplasma gondii (T. gondii) and diabetes mellitus. We perform an age- and gender-matched case-control study to determine the association of $T$. gondii infection and diabetes mellitus.

Methods: Cases included 156 patients with diabetes mellitus and 156 controls without diabetes mellitus who attended in two public clinics in Durango City, Mexico. Sera of cases and controls were tested for the presence of anti-Toxoplasma IgG and IgM antibodies using commercially available enzyme-linked fluorescence assays (ELFA).

Results: Anti-T. gondii IgG antibodies were found in $10(6.4 \%)$ of the 156 cases and in five (3.2\%) of the 156 controls (odds ratio (OR): 2.06; 95\% confidence interval $(\mathrm{CI}): 0.69-6.19 ; \mathrm{P}=0.18)$. The frequency of high $(>150 \mathrm{IU} / \mathrm{mL})$ anti- $T$. gondii $\mathrm{IgG}$ levels in seropositive cases $(1 / 10: 10.0 \%)$ was comparable to the one $(1 / 5: 20 \%)$ in seropositive controls (OR: $0.44 ; 95 \%$ CI: $0.02-9.03 ; \mathrm{P}=1.00$ ). None of the 10 cases and five controls with seropositivity to anti-T. gondii IgG antibodies were positive for anti-T. gondii IgM antibodies. Stratification by gender showed similar frequencies of $T$. gondii infection in female cases (7/107: 6.5\%) and female controls (4/107: 3.7\%) (OR: 1.80; 95\% CI: $0.51-6.34 ; \mathrm{P}=0.53)$, and in male cases $(3 / 49: 6.1 \%)$ and
\end{abstract}

Manuscript accepted for publication April 05, 2017

aFaculty of Medicine and Nutrition, Juarez University of Durango State, Avenida Universidad S/N, 34000 Durango, Dgo, Mexico

bInstitute for Scientific Research "Dr. Roberto Rivera Damm", Juarez University of Durango State, Avenida Universidad S/N, 34000 Durango, Durango, Mexico

${ }^{\mathrm{c}}$ Clinica de Diabetes, Secretaria de Salud, Predio Canoas S/N, 34000 Durango, Dgo, Mexico

${ }^{\mathrm{d}}$ Hospital General de Durango, Secretaria de Salud, Avenida 5 de febrero y Norman Fuentes, 34000 Durango, Dgo, Mexico

${ }^{e}$ Centro de Salud de Servicios Ampliados 450, Toma de Zacatecas 129, 34000 Durango, Dgo, Mexico

${ }^{f}$ Corresponding Author: Cosme Alvarado-Esquivel, Laboratorio de Investigacion Biomedica, Facultad de Medicina y Nutricion, Avenida Universidad S/N, 34000 Durango, Dgo, Mexico. Email: alvaradocosme@yahoo.com

doi: https://doi.org/10.14740/jocmr3029w male controls (1/49: 2.0\%) (OR: 3.13; 95\% CI: $0.31-31.19 ; \mathrm{P}=0.61)$.

Conclusions: We conclude that there is not serological evidence of an association between $T$. gondii infection and diabetes mellitus in the studied subjects in Durango City, Mexico. Further studies to elucidate the role of $T$. gondii in diabetes should be conducted.

Keywords: Toxoplasma gondii; Seroprevalence; Diabetes mellitus; Case-control study

\section{Introduction}

Toxoplasma gondii (T. gondii) is a coccidian parasite causing infections all around the world [1]. There are two main routes of T. gondii transmission to humans: ingestion of food or water contaminated with oocysts shed by $T$. gondii infected cats, and eating raw or undercooked meat containing tissue cysts $[2,3]$. Vertical transmission may also occur when a primary infection is acquired during pregnancy [2,4]. Most T. gondii infections are asymptomatic [5]. Some infected individuals may develop clinical manifestations of toxoplasmosis including lymphadenopathy, chorioretinitis, and meningoencephalitis $[2,5,6]$. A reactivation of a $T$. gondii infection in immunocompromised patients may lead to a life-threatening disease with involvement of the central nervous system [2,5]. After infection, T. gondii spreads to many organs of the host [7]. The presence of $T$. gondii infection in pancreas has been reported in humans and animals. In humans, $T$. gondii infection may cause pancreatitis [8]. In a series of 18 autopsy cases of acquired toxoplasmosis in New York City, three cases had dissemination of T. gondii to pancreas [9]. In fatal toxoplasmic pancreatitis in AIDS patients, autopsies demonstrated pancreatic necrosis with free parasitic forms or tachyzoites [10], and cysts [11]. In animals, fatal acute toxoplasmosis involving pancreas has been observed in experimentally infected mice [12], a naturally infected Valley quail [13], a sand fox [14], and 11 sugar gliders [15].

It is unclear whether involvement of pancreas during $T$. gondii infection may lead to diabetes mellitus. In a Korean study, diabetes mellitus was a major coincidental disease in 
T. gondii IgG seropositive patients [16]. In contrast, significantly lower levels of antibodies against $T$. gondii were found in Colombian patients with type 1 diabetes mellitus [17]. In a prospective cohort of Latino elderly in the USA, individuals seropositive to $T$. gondii did not show an increased rate of diabetes [18]. In an age- and gender-matched case-control study of 91 diabetic cases and 93 healthy non-diabetic controls in Iran, researchers found a higher seroprevalence of IgG antibodies against $T$. gondii in diabetic patients than in healthy controls [19]. In a meta-analysis of studies on the association between chronic toxoplasmosis and diabetes mellitus, researchers found that chronic toxoplasmosis was a possible risk factor for type 2 diabetes mellitus, and no association between T. gondii and type 1 diabetes mellitus [20]. Since results of studies about the association of $T$. gondii infection and diabetes mellitus are controversial, we decided to perform a matched case-control study to determine whether $T$. gondii seropositivity is associated with diabetes mellitus in subjects attending in a public health institution in Durango City, Mexico.

\section{Materials and Methods}

\section{Study design and populations studied}

We performed an age- and gender-matched case-control study of 156 patients with diabetes mellitus and 156 individuals without diabetes mellitus. Patients were enrolled in a public clinic for diabetes care (Clinica de Diabetes), and controls were enrolled in a public health center (Centro de Salud de Servicios Ampliados 450) in Durango City, Mexico. Both clinics belong to the same health institution (Secretaria de Salud). This study was performed from February 2015 to March 2017. Inclusion criteria for enrollment of cases were: 1) individuals with diabetes mellitus attending in a public diabetes care center (Clinica de Diabetes) in Durango City, Mexico; 2) aged 18 years and older; and 3 ) who accepted to participate in the study. Gender, occupation and socioeconomic status were not restrictive criteria for enrollment. In total, 107 (68.6\%) females and 49 (31.4\%) males with diabetes mellitus were enrolled in the study. Mean age in cases was $56.0 \pm 11.1$ (range $18-83$ ) years old. Of the 156 patients, 151 had type II diabetes and five had type I diabetes. Control individuals were matched with cases for age and gender. Control individuals were randomly selected. Inclusion criteria for enrollment of controls were: 1) individuals without diabetes mellitus attending in public health center of the Secretary of Health (Centro de Salud de Servicios Ampliados 450); 2) aged 18 years and older; and 3) who accepted to participate in the study. The control group included $107(68.6 \%)$ females and $49(31.4 \%)$ males. Mean age in control subjects was $55.5 \pm 11.8$ (range $20-85$ ) years old. Cases and controls had similar age $(\mathrm{P}=0.70)$.

\section{Detection of anti- $T$. gondii IgG and IgM antibodies}

Serum samples from cases and controls were obtained and kept frozen at $-20{ }^{\circ} \mathrm{C}$ until analyzed. Serum samples were analyzed for anti-T. gondii IgG antibodies using the commercially available enzyme-linked fluorescent assay (ELFA) kit "VIDAS Toxo IgG II" (BioMerieux, Marcy-l'Etoile, France). All serum samples with positive results in the IgG ELFA were further tested for anti-T. gondii IgM antibodies using the commercially available ELFA kit "VIDAS Toxo IgM" (BioMerieux, Marcy-l'Etoile, France). Both IgG and IgM ELFA were performed following the manufacturer's instructions.

\section{Statistical analysis}

Analysis of data was performed using the software Epi Info 7 and SPSS 15.0 (SPSS Inc., Chicago, IL). For the sample size calculation, we used the following values: a 95\% confidence level, a power of $80 \%$, a 1:1 proportion of cases and controls, and a reference seroprevalence of $6.1 \%$ [21] as the expected frequency of exposure in controls. Thus, a sample size of 155 cases and 155 controls was obtained. We used the Student's $t$-test to compare age values among cases and controls. The association between $T$. gondii infection and diabetes mellitus was analyzed with the two-tailed Pearson's Chi-squared test or the Fisher exact test (for small values). Odds ratios (ORs) and 95\% confidence intervals (CIs) were calculated, and statistical significance was set at a $\mathrm{P}$ value less than 0.05 .

\section{Ethics aspects}

The Ethics Committee of the General Hospital of the Secretary of Health in Durango City, Mexico approved this study. Participation in the study was voluntary, and a written informed consent was obtained from all participants.

\section{Results}

Anti-T. gondii $\operatorname{IgG}$ antibodies were found in 10 (6.4\%) of the 156 cases and in five $(3.2 \%)$ of the 156 controls. The seroprevalence of $T$. gondii infection in cases was similar to the one in controls (OR: 2.06; 95\% CI: $0.69-6.19 ; \mathrm{P}=0.18$ ). Of the 10 anti-T. gondii IgG positive cases, one (10.0\%) had IgG levels higher than $150 \mathrm{IU} / \mathrm{mL}$, and nine (90.0\%) between 8 and 99 $\mathrm{IU} / \mathrm{mL}$. Whereas, of the five anti-T. gondii IgG positive controls, one $(20.0 \%)$ had IgG levels higher than $150 \mathrm{IU} / \mathrm{mL}$, and four $(80.0 \%)$ between 8 and $99 \mathrm{IU} / \mathrm{mL}$. The frequency of high $(>150 \mathrm{IU} / \mathrm{mL})$ anti-T. gondii IgG levels in cases was similar to the one in controls (OR: 0.44; 95\% CI: $0.02-9.03$; $\mathrm{P}=1.00$ ). None of the 10 cases and five controls with seropositivity to anti-T. gondii IgG antibodies were positive for anti-T. gondii IgM antibodies by ELFA. Stratification by gender showed similar frequencies of $T$. gondii infection in female cases $(7 / 107$ : 6.5\%) and female controls (4/107: 3.7\%) (OR: 1.80; 95\% CI: $0.51-6.34 ; \mathrm{P}=0.53)$. The frequency of $T$. gondii infection in male cases (3/49: 6.1\%) was similar to the one in male controls (1/49: 2.0\%) (OR: 3.13; 95\% CI: $0.31-31.19$; P = 0.61). Of the 151 patients with type II diabetes, nine $(6.0 \%)$ were positive to IgG against $T$. gondii, whereas one $(20 \%)$ of the five patients 
with type I diabetes had $\operatorname{IgG}$ antibodies against $T$. gondii $(\mathrm{P}=$ $0.28)$.

\section{Discussion}

Whether $T$. gondii infection is associated with diabetes mellitus is still a matter of controversy. A limited number of studies about this association exist, and have reported conflicting results. Therefore, we sought to determine whether $T$. gondii infection is associated with diabetes mellitus in a sample of adult patients attending in a public clinic for diabetes care in Durango City, Mexico. For this purpose, we assessed the frequency of $\operatorname{IgG}$ and $\operatorname{IgM}$ antibodies against $T$. gondii, and the anti-T. gondii IgG antibody levels. Results of the current study indicate that patients with diabetes mellitus have equal frequencies of $\operatorname{IgG}$ and $\operatorname{IgM}$ antibodies against $T$. gondii, and anti-T. gondii IgG antibody levels as age- and gender-matched control subjects without diabetes. Therefore, our findings based on serological methods do not support an association between diabetes mellitus and $T$. gondii infection. Our results agree with the low $(9 \%)$ seroprevalence of $T$. gondii infection reported in Mexican Americans from San Antonio Texas, USA that suffered from high rates of obesity and type 2 diabetes mellitus [22], and with low levels of antibodies against $T$. gondii found in Colombian patients with type 1 diabetes mellitus [17]. In addition, our results are in line with the lack of association between $T$. gondii infection and incident diabetes found in a prospective cohort of Latino elderly in New York, USA [18]. On the other hand, findings in favor of an association between T. gondii seropositivity and diabetes mellitus include a high seroprevalence of $T$. gondii infection found in patients with diabetes mellitus in general hospitals in Daejeon, Korea [16], and a significantly higher seroprevalence of $T$. gondii infection in patients with diabetes than in healthy controls found in an age- and gender-matched case-control study in Iran [19]. In addition, a meta-analysis of seven studies about the association of chronic toxoplasmosis and diabetes mellitus concluded that chronic toxoplasmosis was a possible risk factor for type 2 diabetes mellitus [20].

For a fair comparison of our results obtained under an ageand gender-matched case-control study design, we searched for similar studies in the medical literature. We were able to find only one age- and gender-matched case-control study about the association of T. gondii seropositivity and diabetes mellitus. In such study, researchers found a positive association between $T$. gondii infection and diabetes in Iran [19]. It is not clear why there were differences in the associations among the studies. It is likely that differences in the characteristics of the studied populations among the studies might explain the differences in the association. For instance, differences in age and gender among the studied populations might influence the seroprevalence of $T$. gondii infection. We could not compare the age and gender variables of our studied population with those of the Iranian study because no description of these variables was found in the Iranian study. A clear difference among the studies was the laboratory methods used. We used ELFA to determine antibodies against $T$. gondii whereas an enzyme- linked immunosorbent assay was used in the Iranian study [19]. Sensitivity and specificity of the IgG tests used in the studies are comparable. A $100 \%$ sensitivity and a $99 \%$ specificity of the enzyme-linked immunosorbent assay used were reported in the Iranian study [19]. According to the insert, the ELFA used in our study has a $99.65 \%$ sensitivity and a $99.92 \%$ specificity. The numbers of cases and controls were different among the studies. We enrolled 156 cases and 156 controls whereas 91 cases and 93 controls were included in the Iranian study [19].

The present study has limitations: we studied only diabetic outpatients from a single public clinic for diabetes care. The great majority of patients attending in this clinic have low socioeconomic status. The severity of diabetes in outpatients may be milder than in inpatients. Therefore, additional studies with diabetic inpatients, of diverse socioeconomic status, in several clinics should be conducted to further determine the association between $T$. gondii infection and diabetes mellitus.

\section{Conclusions}

We conclude that there was not serological evidence of an association between $T$. gondii infection and diabetes in adult patients attending in a public diabetes care center in Durango City, Mexico. Further studies to elucidate the role of $T$. gondii in diabetes should be conducted.

\section{Financial Support}

This study was financially supported by Juarez University of Durango State, Mexico.

\section{Competing Interests}

The authors declare that no competing interests exist.

\section{References}

1. Battiprolu PK, Gillette TG, Wang ZV, Lavandero S, Hill JA. Diabetic Cardiomyopathy: Mechanisms and Therapeutic Targets. Drug Discov Today Dis Mech. 2010;7(2):e135-e143.

2. Montoya JG, Liesenfeld O. Toxoplasmosis. Lancet. 2004;363(9425):1965-1976.

3. Jones JL, Dubey JP. Foodborne toxoplasmosis. Clin Infect Dis. 2012;55(6):845-851.

4. Hampton MM. Congenital Toxoplasmosis: A Review. Neonatal Netw. 2015;34(5):274-278.

5. Saadatnia G, Golkar M. A review on human toxoplasmosis. Scand J Infect Dis. 2012;44(11):805-814.

6. Pradhan S, Yadav R, Mishra VN. Toxoplasma meningoencephalitis in HIV-seronegative patients: clinical patterns, imaging features and treatment outcome. Trans $\mathrm{R}$ Soc Trop Med Hyg. 2007;101(1):25-33. 
7. Harker KS, Ueno N, Lodoen MB. Toxoplasma gondii dissemination: a parasite's journey through the infected host. Parasite Immunol. 2015;37(3):141-149.

8. Parenti DM, Steinberg W, Kang P. Infectious causes of acute pancreatitis. Pancreas. 1996;13(4):356-371.

9. Liu D, Ling Q, Venkataseshan VS. [A clinicopathological study of eighteen autopsy cases with acquired toxoplasmosis]. Zhongguo Ji Sheng Chong Xue Yu Ji Sheng Chong Bing Za Zhi. 1995;13(1):64-67.

10. Hofman P, Michiels JF, Mondain V, Saint-Paul MC, Rampal A, Loubiere R. [Acute toxoplasmic pancreatitis. An unusual cause of death in AIDS]. Gastroenterol Clin Biol. 1994;18(10):895-897.

11. Ahuja SK, Ahuja SS, Thelmo W, Seymour A, Phelps KR. Necrotizing pancreatitis and multisystem organ failure associated with toxoplasmosis in a patient with AIDS. Clin Infect Dis. 1993;16(3):432-434.

12. Sukthana Y, Waree P, Pongponratn E, Chaisri U, Riganti M. Pathologic study of acute toxoplasmosis in experimental animals. Southeast Asian J Trop Med Public Health. 2003;34(1):16-21.

13. Casagrande RA, Pena HF, Cabral AD, Rolim VM, de Oliveira LG, Boabaid FM, Wouters AT, et al. Fatal systemic toxoplasmosis in Valley quail (Callipepla californica). Int J Parasitol Parasites Wildl. 2015;4(2):264-267.

14. Pas A, Dubey JP. Toxoplasmosis in sand fox (Vulpes rueppelli). J Parasitol. 2008;94(4):976-977.

15. Barrows M. Toxoplasmosis in a colony of sugar gliders (Petaurus breviceps). Vet Clin North Am Exot Anim Pract. 2006;9(3):617-623.

16. Shin DW, Cha DY, Hua QJ, Cha GH, Lee YH. Seropreva- lence of Toxoplasma gondii infection and characteristics of seropositive patients in general hospitals in Daejeon, Korea. Korean J Parasitol. 2009;47(2):125-130.

17. Krause I, Anaya JM, Fraser A, Barzilai O, Ram M, Abad $\mathrm{V}$, Arango A, et al. Anti-infectious antibodies and autoimmune-associated autoantibodies in patients with type I diabetes mellitus and their close family members. Ann N Y Acad Sci. 2009;1173:633-639.

18. Jeon CY, Haan MN, Cheng C, Clayton ER, Mayeda ER, Miller JW, Aiello AE. Helicobacter pylori infection is associated with an increased rate of diabetes. Diabetes Care. 2012;35(3):520-525.

19. Shirbazou S, Delpisheh A, Mokhetari R, Tavakoli G. Serologic Detection of Anti Toxoplasma gondii Infection in Diabetic Patients. Iran Red Crescent Med J. 2013;15(8):701-703.

20. Majidiani H, Dalvand S, Daryani A, Galvan-Ramirez ML, Foroutan-Rad M. Is chronic toxoplasmosis a risk factor for diabetes mellitus? A systematic review and meta-analysis of case-control studies. Braz J Infect Dis. 2016;20(6):605-609.

21. Alvarado-Esquivel C, Estrada-Martinez S, Pizarro-Villalobos H, Arce-Quinones M, Liesenfeld O, Dubey JP. Seroepidemiology of Toxoplasma gondii infection in general population in a northern Mexican city. J Parasitol. 2011;97(1):40-43.

22. Rubicz R, Leach CT, Kraig E, Dhurandhar NV, Grubbs B, Blangero J, Yolken R, et al. Seroprevalence of 13 common pathogens in a rapidly growing U.S. minority population: Mexican Americans from San Antonio, TX. BMC Res Notes. 2011;4:433. 